\title{
Physically Realistic Roughness Closure Scheme to Simulate Turbulent Channel Flow over Rough Beds within the Framework of LES
}

\author{
T. Stoesser, M.ASCE ${ }^{1}$
}

\begin{abstract}
A physically realistic roughness closure method for the simulation of turbulent open-channel flow over natural beds within the framework of large-eddy simulation (LES) is proposed. The description of bed roughness in LES is accomplished through a roughness geometry function together with forcing terms in the momentum equations. The major benefit of this method is that the roughness is generated from one physically measurable parameter, i.e., the mean grain diameter of the bed material. A series of flows over rough beds, for which mean flow and turbulence statistics are available from experiments, is simulated. Measured and computed values are compared to validate the proposed roughness closure approach. It is found that predicted streamwise velocity profiles, turbulence intensities, and turbulent shear stress profiles match the measured values fairly well. Furthermore, the effect of roughness on the overall flow resistance is predicted in reasonable agreement with experimental values.
\end{abstract}

DOI: 10.1061/(ASCE)HY.1943-7900.0000236

CE Database subject headings: Channel flow; River beds; Turbulence; Bed roughness.

Author keywords: LES; Rough bed; Turbulence; Roughness closure.

\section{Introduction}

Turbulent flows and the associated transport processes in streams, rivers, and estuaries are strongly influenced by the roughness conditions on the channel bed. The characteristics of the roughness geometry can vary significantly and particularly in alluvial channels researchers and engineers face high spatial heterogeneity of the channel-bed roughness. In a layer near the bed, known as the roughness layer, the flow is directly influenced by the individual roughness elements and is therefore spatially highly inhomogeneous; i.e., time-averaged statistics are not independent of location, at the same mean wall-normal distance. Through spatial averaging over a predefined area (significantly larger than the size of the individual roughness elements) a representative velocity profile within the roughness sublayer and the adjoining overlap layer can be formulated (Nikora et al. 2001, 2004, 2007). However, the formulation includes (empirical and nonuniversal) parameters, which seem to depend on the roughness geometry, the relative submergence, and other factors, and it is difficult to estimate these parameters a priori. Moreover, formal spatial averaging introduces new "form-induced" stresses that are contributors to the momentum flux balance. The quantification of these dispersive stresses is currently a subject of ongoing research.

Numerical simulations have so far not significantly advanced the understanding of flows over rough beds as to date most simu-

${ }^{1}$ School of Civil and Environmental Engineering, Georgia Institute of Technology, Atlanta, GA 30332 (corresponding author). E-mail: thorsten@ce.gatech.edu

Note. This manuscript was submitted on August 1, 2009; approved on March 27, 2010; published online on March 30, 2010. Discussion period open until March 1, 2011; separate discussions must be submitted for individual papers. This technical note is part of the Journal of Hydraulic Engineering, Vol. 136, No. 10, October 1, 2010. @ASCE, ISSN 07339429/2010/10-812-819/\$25.00. lations have been based on the Reynolds-averaged Navier-Stokes (RANS) equations, in which the effect of roughness has been accounted for by roughness functions determined empirically from experiments. The success of direct numerical simulations (DNSs) and large-eddy simulations (LESs) in revealing details of the turbulent flow over smooth walls has initiated DNS and LES studies of flow over rough walls. As the numerical effort for DNS or LES is considerably higher than for RANS simulations only very few studies have been reported to date. Most DNSs or LESs were performed for flows over exactly defined "roughness elements" such as square bars (e.g., Leonardi et al. 2003; Stoesser and Nikora 2008), wavy walls (e.g., Calhoun and Street 2001; Nakayama et al. 2004), or typical bed forms such as sand dunes (e.g., Yue et al. 2006; Stoesser et al. 2008) and provided an enormous wealth of statistical data and insight into turbulence structures. While these simulations resolved the roughness explicitly through the numerical grid, the virtual boundary method was used by Lee (2002), Cui et al. (2003), and Bhaganagar et al. (2004) as a less expensive alternative. In the virtual boundary method the rough wall, composed of individual, geometrically predefined roughness elements, is embedded in a Cartesian grid, and the no-slip condition on the immersed elements is imposed by appropriate body forces. In these studies the shape and distribution of the roughness elements were known a priori and were resolved by several grid points. However, in open-channel flow over an alluvial bed, the rough surface comprises sediments, the size of which can range from micrometers to decimeters with relative submergences ranging from one to several hundreds. Clearly, currently available computational resources do not allow for resolving every single particle in an alluvial channel; even if the detailed bathymetry of the rough surface was known (which is hardly the case). To overcome these limitations, Nakayama et al. (2004) suggested adding extra dispersive stress terms to the momentum equations and using a slip boundary condition at the virtual boundary to mimic the roughness. This method reproduces the 


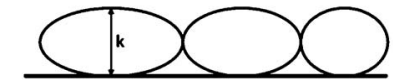

a)
Present Study

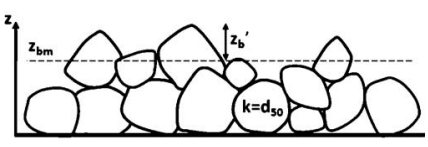

b)
Fig. 1. Sketch of the sandpaper roughness by Scotti (2006) and the alluvial rough bed as generated in the present study

flow statistics fairly well but requires a priori knowledge of the magnitude of the dispersive stresses, which Nakayama et al. (2004) determined from a DNS. Probably the most intriguing approach to date to model roughness within the framework of DNS/ LES was recently suggested by Scotti (2006). He proposed to mimic sandpaper roughness by randomly placing ellipsoids of height $k$ on a smooth wall. Scotti (2006) accounted for the ellipsoids in the momentum equations through a porosity approach and reproduced the downward shift of the velocity on a Clauser plot as well as realistic turbulence statistics. In here, Scotti's (2006) sandpaper roughness method is extended to natural channel-bed roughness. Rough beds of four selected testcases are generated with a roughness geometry function. High resolution LESs are then performed and first- and second-order statistics are compared with laboratory data to validate the method.

\section{Generation of Natural Channel-Bed Roughness}

The roughness closure method proposed should be regarded a modification or extension of Scotti's (2006) virtual sandpaper method. While Scotti's (2006) approach is designed for technical roughness, i.e., well-defined element shape and uniform roughness height $k$ [see Fig. 1(a)], the roughness closure approach introduced herein mimics a natural, alluvial channel bed [Fig. 1(b)]. The basic idea is to generate a realistic, natural channel-bed topology from a measurable physical parameter of the sediment material of which the bed is composed of. Therefore, a roughness geometry function $A\left(z^{\prime}\right)$ as presented in Nikora et al. (2001) is employed. The function $A\left(z^{\prime}\right)$ describes the cumulative probability distribution of bed elevations $(z)$ for natural, impermeable rough beds. Nikora et al. (2001) compared bathymetric data from water-worked gravel beds of New Zealand rivers and from an unworked gravel bed created manually in a flume and found that the roughness geometry functions $A\left(z^{\prime}\right)$ of both behave similarly.
Furthermore, the distributions are fairly close to the cumulative probability function $(\mathrm{CPF})$ for the normal distribution (Nikora et al. 2001). This finding was later confirmed by Aberle and Nikora (2006) and Aberle (2007) who sampled high resolution digital elevation data of water-worked gravel beds through an accurate laser displacement meter. A slight skewness in the distribution, however, was observed by Aberle and Nikora (2006) for gravel beds that were armored by high discharges before the beds were surveyed.

For the simulations the natural channel bed is initially generated from random numbers with a normal distribution using the polar method of Marsaglia and Bray (1964) through specification of the mean $z_{b m}$ and the standard deviation $\sigma_{z}$ of the bed elevation. The standard deviation of the bed elevation was chosen as $\sigma_{z}=0.5 d_{50}$, which is reasoned by Aberle and Nikora's (2006) data [Fig. 2(a)] suggesting strong correlation between the characteristic grain size $d_{50}$ and the standard deviation $\sigma_{z}$. Fig. 2(b) presents the CPF of the bed roughness created with the above described method for one of the cases modeled in this study (R\&W2). It is apparent that the CPF of the generated $R \& W 2$ bed shows very good correspondence with the CPFs of a water-worked river bed and an unworked flume bed [the data were obtained from Nikora, personal communication, 2007; and is published in Nikora et al. (2001)], in particular, with the unworked bed, as the effect of armoring was not included herein.

\section{Navier-Stokes Solver}

The LES code HYDRO3D-GT (Stoesser and Nikora 2008) is used to perform the LES. The code solves the filtered NavierStokes equations on a Cartesian, block-structured grid using the finite volume method. Both convective and diffusive fluxes are approximated with central differences of second-order accuracy. The SIMPLE algorithm is employed to conserve mass and to couple the pressure to the velocity field. Time advancement is achieved by a second-order explicit Runge-Kutta scheme. The subgrid stresses of the filtered Navier-Stokes equations are computed using the dynamic version (Germano et al. 1991) of the standard Smagorinsky (1963) model. The right-handed coordinate system is implied throughout; i.e., the $x$ axis is oriented along the main flow parallel to the bed ( $u$-velocity component), the $y$ axis is oriented to the left bank ( $v$-velocity component), and the $z$ axis is pointing toward the water surface ( $w$-velocity component), with the origin at the lowest point of the rough channel bed. The bed

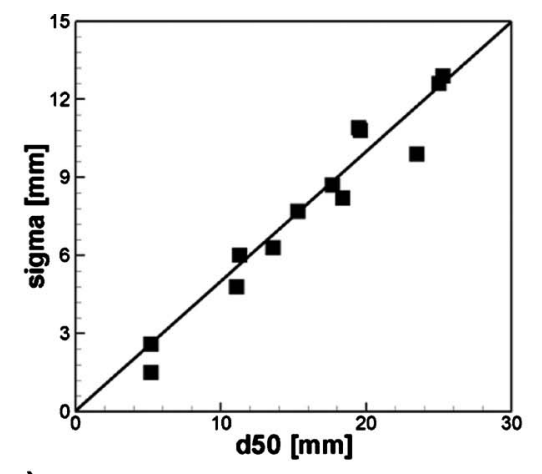

a)

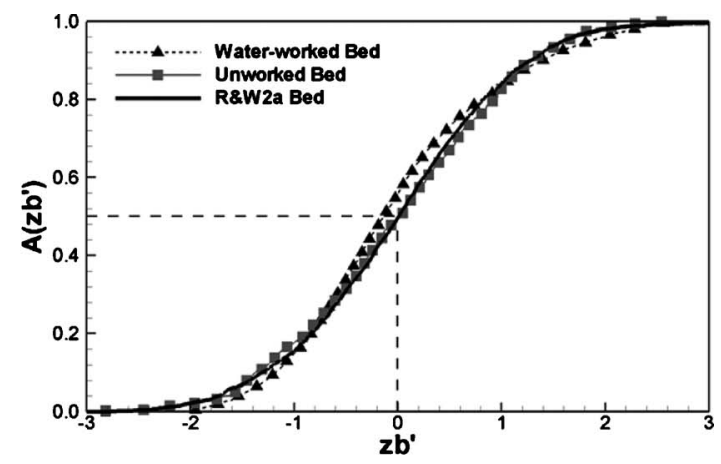

b)

Fig. 2. (a) Correlation between the standard deviation and the characteristic diameter $d_{50}$ of water-worked gravel beds (data from Aberle and Nikora 2006); (b) CPFs of the bed roughness of the D\&W2 case, a water-worked gravel bed and an unworked flume bed (data of water-worked and -unworked beds obtained from Nikora (personal communication, 2007) 
Table 1. Key Dimensionless Parameters of the Four Experiments Selected for Validation

\begin{tabular}{lccc}
\hline & R\&W (2005) & R\&W (2005) & R\&W (2005) \\
$d_{50}=2.5 \mathrm{~mm}$ & $d_{50}=11.5 \mathrm{~mm}$ & $\begin{array}{c}\text { Grass (1971) } \\
d_{50}=21 \mathrm{~mm}\end{array}$ & 2.5 \\
$\mathrm{~mm}$
\end{tabular}

roughness is incorporated by using a forcing term that is added to the right-hand side of the normalized momentum equations. For the $u$-equation this force reads in time-discrete form

$$
f_{u}= \begin{cases}C-D+\nabla P+\frac{\Delta V}{\Delta t}\left(U_{t}-U^{n}\right) & \text { inside the roughness } \\ 0 & \text { elsewhere }\end{cases}
$$

in which $C=$ discrete convection term; $D=$ discrete diffusion term; $P=$ resolved pressure divided by the density; $U^{n}=$ resolved velocity in streamwise direction at the previous time step; and $U_{t}$ $=$ target velocity.

The solution procedure is as follows: (1) solve the momentum equations for convection diffusion, (2) apply forcing term within the bed to force the velocity to a prescribed target velocity (here $\left.U_{t}=0.0\right)$ in all Runge-Kutta steps, and (3) apply the pressure correction to enforce continuity after the last Runge-Kutta step.

The fluid-solid interface is not sharply resolved as in an immersed boundary method, but as Scotti (2006) showed, such an accurate treatment is not necessarily needed, since only spatially averaged statistics of the flow are sought. In other words, profiles of simulated flow statistics may not match at single locations but should collapse reasonable accurate mean profile when spatially averaged over a finite number of locations.

\section{Setup and Boundary Conditions}

Several laboratory experiments of the flow over rough beds are selected in this study, the data of which are used to validate the implementation of the proposed roughness closure scheme in the LES code. Table 1 provides an overview of the flow conditions of the experiments. The roughness length scale $k$ chosen here is the characteristic sediment size $d_{50}$, which is used as the input parameter for generating the rough bed. Three experiments from Rahman and Webster (2005) and one experiment from Grass (1971) are chosen, because the bed material is fairly uniform and $d_{50}$ values from sediment samples are provided. The channel Reynolds number $\mathrm{R}_{h}$, i.e., based on the channel depth and bulk velocity, is relatively constant; i.e., $\mathrm{R}_{h}=7,250$ for Grass' (1971) experiment and $\mathrm{R}_{h}=10,000$ for all three Rahman and Webster (2005) experiments. The Reynolds number based on the friction velocity $u_{*}$ and the water depth $h$ varies between $\mathrm{R}_{\tau}=525$ and $R_{\tau}=1,164$. The greatest variation is found in the grain or roughness Reynolds number (i.e., $\mathrm{R}_{*}=d_{50} u_{*} / \nu=9-122$ ). The computational domain spans $6 h$ in streamwise, $3 h$ in spanwise, and $h$ in vertical directions, respectively. The domain size is believed to be large enough to contain the largest turbulence structures hence avoiding alteration of the flow statistics and is of similar size than the computational box for the smooth wall channel flow LES of
Germano et al. (1991), who used a $2 \pi h x 4 / 3 \pi h x h$, or Scotti (2006) for his transitional roughness DNSs $(6 h x 2 h x h)$. The same grid is used for all four cases and consists of $241 \times 161 \times 121$ grid points. Simulations at a coarser grid resolution were carried out for each case and yielded similar results. In the following, only the results from the finest grid are shown and discussed. The grid is uniformly spaced in both horizontal directions, and the grid spacing in terms of wall units for the fine grid are $\Delta x^{+}$ $=\left(\Delta x \cdot u_{*}\right) / v \approx 10-30$ (depending on the experiment) in the streamwise and $\Delta y^{+}=\left(\Delta y \cdot u_{*}\right) / v \approx 10-20$ in the spanwise direction. In the vertical direction the grid is uniformly spaced for 0 $<z<0.2 h$ with $\Delta z^{+}=\left(\Delta z \cdot u_{*}\right) / v \approx 3-6$ and is then stretched toward the surface. The roughness-height-to-grid-spacing ratio varies from $d_{50} / \Delta z=3$ to $d_{50} / \Delta z=20$. A longitudinal slice of the grid is presented in Fig. 3, in which the generated roughness is embedded (shaded area). The lowest elevation of the roughness is at $z=0.0$. Periodic boundary conditions are applied in the streamwise and spanwise directions. A frictionless rigid lid slip condition is applied at the water surface, which is expected to be flat and parallel to the bed due to the small Froude numbers. The flow is driven by an imposed pressure gradient in the streamwise direction to maintain a constant massflux with $u$ (bulk) $=1.0$. The integral shear velocity and the total wall shear stress used for the normalization of turbulence quantities and Reynolds stresses are unambiguously obtained from the time-averaged pressure gradient; i.e., $\tau_{0}=\langle d p / d x\rangle \cdot h$ and $u_{*}=\left(\tau_{0} / \rho\right)^{1 / 2}$.

The simulation was initially run for approximately 20 flowthrough times $T$, with $T=6 h / u$ (bulk) in order to establish the flow and reach fully developed turbulence. After this initial period the LESs were continued for another 100 +flow-through times during which data were collected to compute the flow statistics.

\section{Results and Discussion}

Fig. 4 presents computed time and space averaged normalized velocity profiles of the selected four validation cases on a semi-

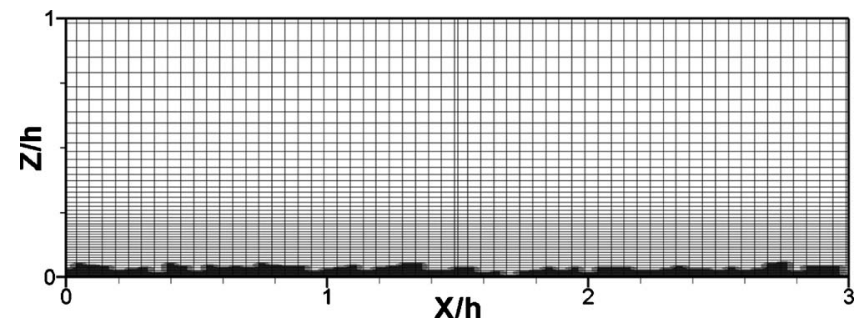

Fig. 3. Longitudinal plane of the numerical grid including the embedded bed roughness (black contour) for the Grass (1971) case; note that only half of the domain and every second grid line are shown 


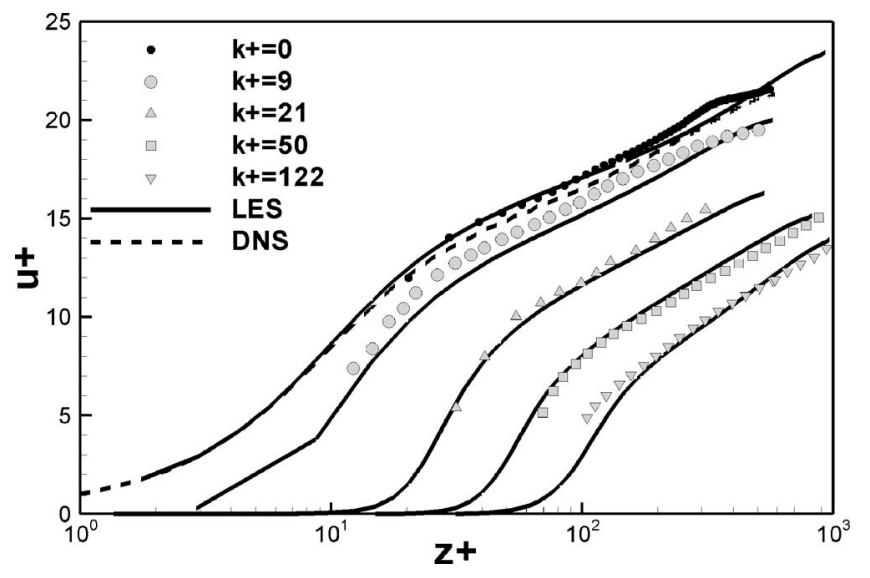

Fig. 4. Velocity profiles in wall coordinates of the four validation cases on a semilogarithmic plot; DNS and LES data of smooth wall flow simulations and experiments are added for comparison

logarithmic Clauser plot. Also plotted are the data of the respective experiments allowing a direct comparison experimentsimulation, with which the proposed roughness closure approaches' performance can be assessed. Additional results from a smooth bed LES, data from one of the most prominent smooth wall channel flow DNS (Moser et al. 1999), and experimental values from Rahman and Webster (2005), for the flow over a smooth bed, are also plotted. Overall, good agreement between simulated and measured velocities is found and maximum differences are detectable near the bed or near the free surface, where local effects (i.e., near the bed where the exact bed geometry is of importance) or supergrid modeling errors (i.e., using a slip boundary condition at the water surface) come into play. The velocity profile of the smallest roughness is relatively close to the profiles over the smooth wall. The rough bed cases are characterized by a parallel downshift of the velocity profiles in the outer layer. This is in line with numerous experimental findings; i.e., the greater the roughness height, the bigger the downshift. The shift of the velocity profiles $\Delta B$ for each case is calculated and provided in Table 2. The empirical relationship by Cebeci and Bradshaw (1977) is then used to estimate the equivalent grain roughness $k_{s}$ of the rough beds. The equivalent grain roughness, normalized with the characteristic sediment diameter $d_{50}$, is increasing for larger grain diameters from $k_{s} / d_{50}=1.3$ to $k_{s} / d_{50}=2.5$. This reflects the fact that when the sediment size increases, individual grains are more exposed and the flow transitions from quasismooth flow toward wake interference flow. The roughness Reynolds number based on the equivalent grain roughness $k_{s}$ varies between $k_{s}^{+}=10$ and $k_{s}^{+}=307$.

Fig. 5 compares measured and calculated normalized turbulent shear stress profiles of the four validation cases. The LES profiles are time and space averaged and exhibit the linear decrease of the turbulent shear stress toward the water surface. The influence of roughness on the shear stress profile is visible near the bed where pressure drag forces dominate the momentum fluxes. The agreement between simulated and measured shear stresses is best for the $k^{+}=50$ case; however, there is quite some scatter and some unphysical kinks in the experimental data, in particular, for $k^{+}$ $=9$ and $k^{+}=122$. These kinks reflect the uncertainty in determining the integral shear stress $\tau_{0}$ in physical experiments of flow over a natural bed, where local effects become a factor. The predicted integral shear velocity $u_{*}$ is compared with measured values in Fig. 6. As the scatter plot in Fig. 6(a) illustrates, predictions are in reasonably good agreement with measurements. The maximum difference is found to be around $15 \%$ for the bed with the largest grain diameter [Fig. 6(b)]. However, the match or mismatch, respectively, of predicted versus measured global shear velocities is quite well reflected in the shear stress profiles of Fig. 6.

Fig. 7 compares calculated with measured streamwise and vertical turbulence intensities for the four validation cases. The overall agreement is reasonably good for all cases underlining the suitability of the roughness closure method to be able to capture the relevant physical mechanisms in the roughness layer. The streamwise turbulence intensities seem to agree somewhat better with the experiments than the vertical turbulence intensity profiles, for which the experimental data are supposed to be less reliable (D. Webster, personal communication, 2009). As the grain size increases, the variation in bed elevation is more pronounced and local recirculation zones behind exposed grains are larger, and separated vortices interfere with each other. What follows is not only an increase in global bed-shear stress but also a thicker roughness layer and more uniform distribution of streamwise turbulence intensities over the flow depth, hence a decrease in peak turbulence intensity. This is further illustrated in Fig. 8, in which the near bed peak streamwise turbulence intensity is plotted as a function of relative roughness height. In Fig. 8 values from a number of rough bed open-channel flow experiments (Bayazit 1975; Carollo et al. 2005; Dittrich and Koll 1997; Kironoto and Graf 1994; Nikora et al. 2001; Nikora and Goring 2000; Song et al. 1994; Tachie et al. 2000, 2004; Wang et al. 1993, 1996), as well as from recent DNSs (Scotti 2006; Singh et al. 2007), are plotted for comparison. A similar figure was provided by Lamb et al. (2008), who suggested a linear relationship for the peak turbulence intensity (solid black line in Fig. 8). The present LES predictions (black circles) follow the observed trend remarkably well.

Fig. 9(a) presents a snapshot of contours of the streamwise turbulent fluctuation $u$ in a longitudinal plane of half of the domain for the roughest case. Figs. 9(b and c) show perturbation vectors for the $k^{+}=50$ and $k^{+}=122$ cases. Most of the turbulence is produced from local flow separation and recirculation behind exposed sediments. The advantage of the proposed method in contrast to wall functions for rough beds is that such important features of the flow in the roughness layer can be reproduced by the proposed approach without having to know the exact geom-

Table 2. Dimensionless Parameters Obtained from the Simulations

\begin{tabular}{|c|c|c|c|c|}
\hline & $\begin{array}{l}\mathrm{R} \& \mathrm{~W}(2005) \\
d_{50}=2.5 \mathrm{~mm}\end{array}$ & $\begin{array}{c}\mathrm{R} \& \mathrm{~W}(2005) \\
d_{50}=11.5 \mathrm{~mm}\end{array}$ & $\begin{array}{c}\mathrm{R} \& \mathrm{~W}(2005) \\
d_{50}=21 \mathrm{~mm}\end{array}$ & $\begin{array}{c}\text { Grass (1971) } \\
d_{50}=2 \mathrm{~mm} \\
\end{array}$ \\
\hline Roughness coefficient, $\Delta B$ & 1.5 & 8.02 & 10.5 & 4.8 \\
\hline Equivalent sandgrain roughness, $k_{s} / d_{50}$ & 1.3 & 1.91 & 2.47 & 1.8 \\
\hline Relative submergence, $h / k_{s}$ & 61.5 & 9.1 & 3.8 & 13.9 \\
\hline Roughness height, $k_{s}^{+}=\left(u_{*} \cdot k_{s}\right) / v$ & 10 & 92 & 257 & 33 \\
\hline
\end{tabular}



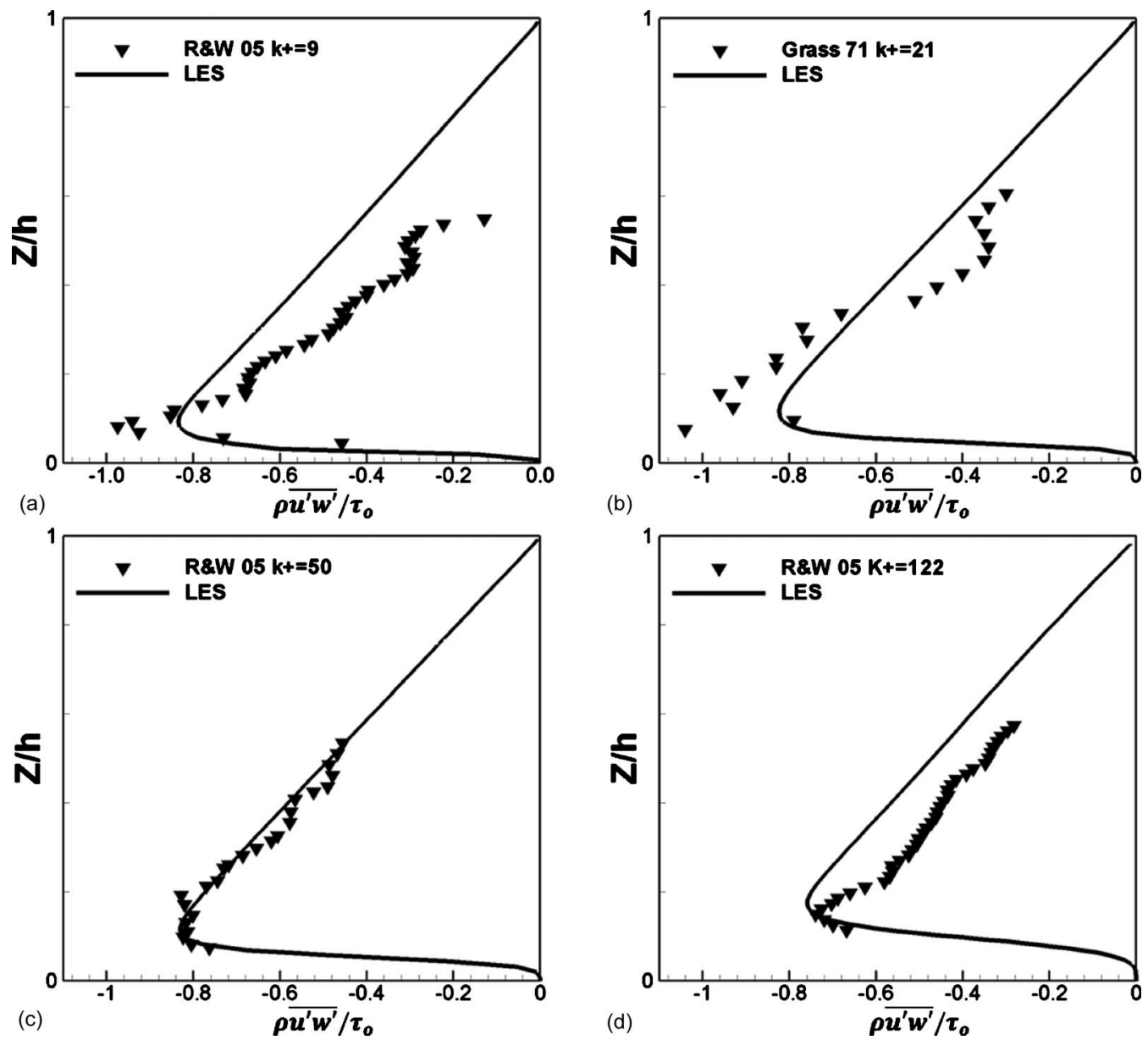

Fig. 5. Vertical distribution of calculated and measured normalized shear stresses of the four cases: (a) R\&WI; (b) Grass; (c) R\&WII; and (d) R\&WIII

etry of the bed. Even though the simulated near wall flow is not exactly the same as in the experiment (in a local sense), above comparisons show that the spatially averaged flow statistics match observations remarkably well. This can be considered a major step forward in the treatment of natural roughness in high resolution wall-resolving LESs or DNSs.

\section{Conclusion}

A physically realistic method for the simulation of turbulent openchannel flow over natural bed roughness within the framework of LES has been proposed and validated. A roughness geometry function that statistically describes bed roughness of water-

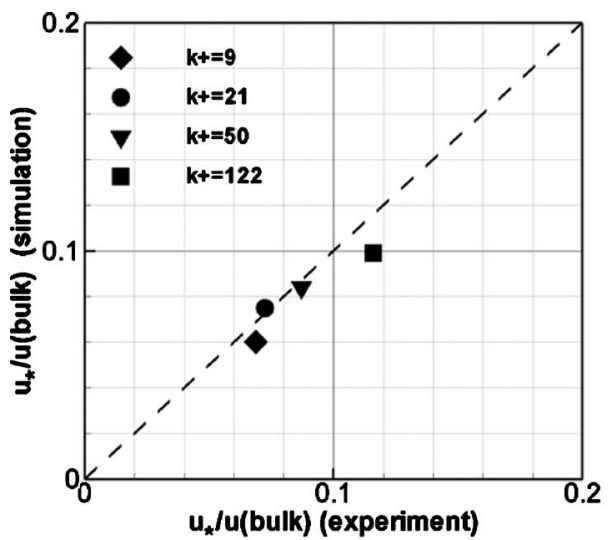

a)

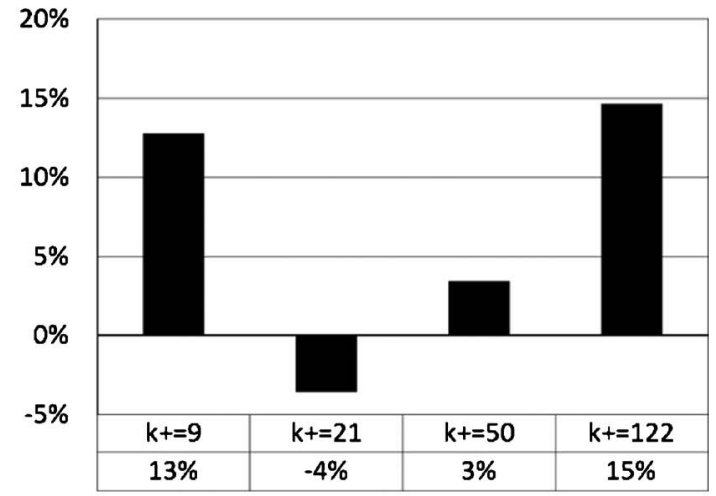

b)

Fig. 6. (a) Correlation between experimental and simulated dimensionless shear velocities; (b) differences between measured and simulated global shear velocities in percent 

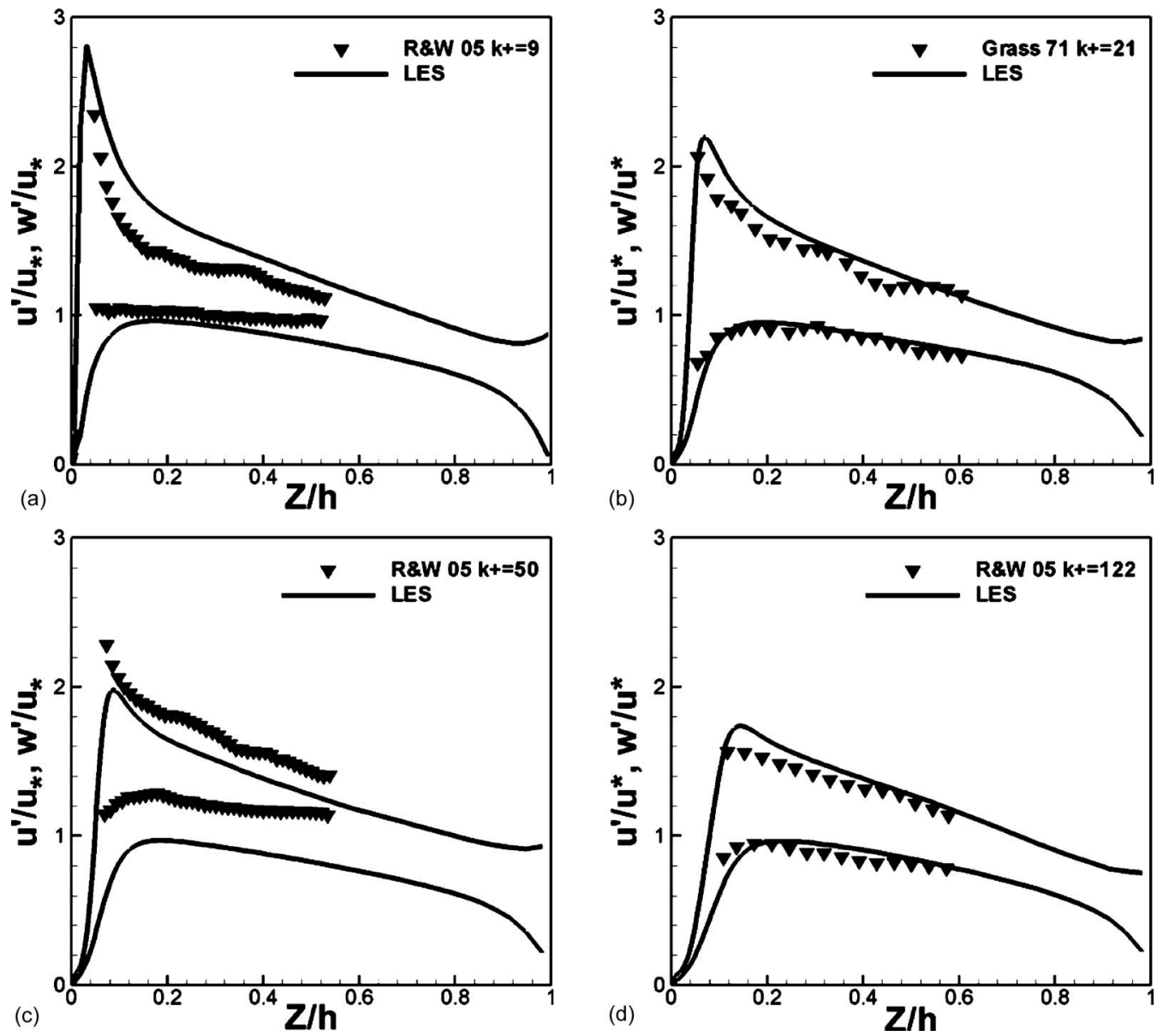

Fig. 7. Distribution of calculated and measured streamwise and wall-normal turbulence intensities of the four cases: (a) R\&WI; (b) Grass; (c) R\&WII; and (d) R\&WIII

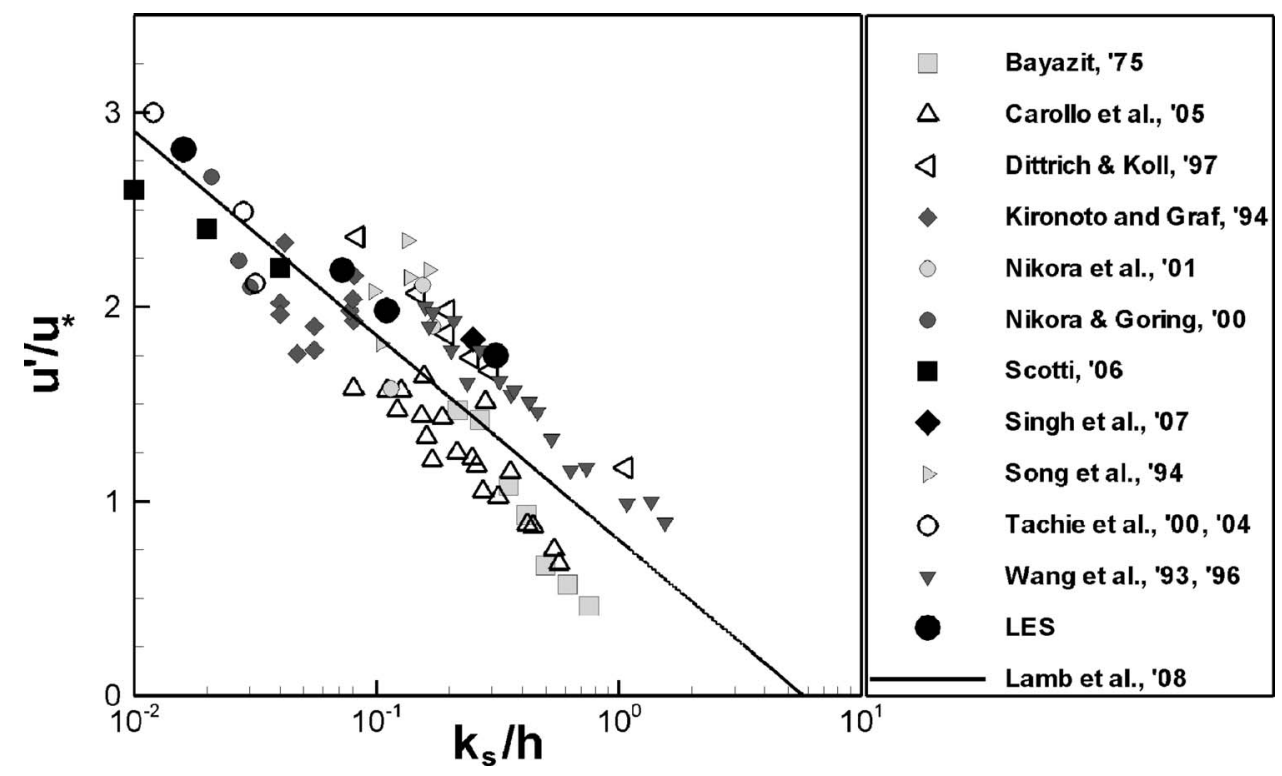

Fig. 8. Peak turbulence intensity as a function of relative submergence for flow over rough beds from the LES, experiments, and DNSs; the solid line is the model fit to the data as suggested by Lamb et al. (2008) 

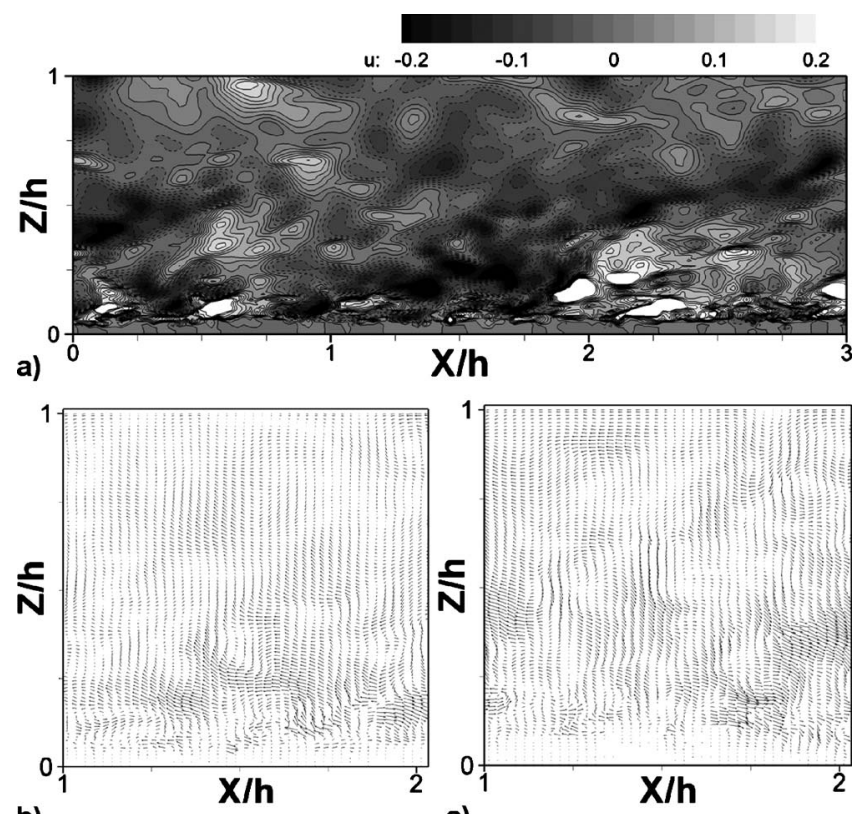

c)

Fig. 9. (a) Contours of the instantaneous streamwise velocity fluctuation for the $k^{+}=50$ case in a longitudinal plane; (b) snapshots of the perturbation velocity vector for the $k^{+}=50$ case; and (c) for the $k^{+}=122$ case

worked sand and gravel beds has been implemented into a LES code. The proposed method requires only one, physically measurable, parameter with which the rough bed is generated. Simulations of flow over rough beds at varying relative submergence were performed and were compared with experimental data to validate the method. Overall, reasonably good agreement is found regarding the streamwise velocity profile, as well as shear stresses and turbulence intensities. The effect of the bed roughness on the overall flow resistance is predicted reasonably accurate and deviations can be regarded within the margins of error in determining such quantities in rough bed flows. Near bed turbulence production is driven by sediment grain exposure, reducing the peak of the streamwise turbulence intensities or flattening the streamwise turbulence intensity profile, respectively. This trend is reproduced remarkably well with the proposed method.

\section{Acknowledgments}

Provision of data by Professor D. Webster (Georgia Institute of Technology), Professor V. Nikora (University of Aberdeen), and Dr. J. Aberle (University of Braunschweig) is gratefully acknowledged.

\section{Notation}

The following symbols are used in this technical note:

$$
\begin{aligned}
A\left(z^{\prime}\right) & =\text { roughness geometry function; } \\
C & =\text { discrete convective term; } \\
D & =\text { discrete diffusion term; } \\
d_{50} & =\text { characteristic grain size; } \\
f & =\text { forcing term; } \\
h & =\text { flow depth; } \\
k & =\text { roughness height; }
\end{aligned}
$$

$P=$ resolved pressure divided by the density;

$\mathrm{R}=$ Reynolds number;

$T=$ flow-through time;

$U=$ resolved velocity;

$\langle U\rangle=$ angular brackets denote time and space averaging;

$u=$ streamwise velocity fluctuation;

$u_{*}=$ friction velocity;

$u($ bulk $)=$ bulk velocity;

$u^{\prime} / u_{*}=$ streamwise turbulent intensity;

$w^{\prime} / u_{*}=$ vertical turbulence intensity;

$z^{+}=$superscript + indicates wall units;

$\Delta x, \Delta y, \Delta z=$ mesh sizes in each direction;

$\kappa=$ von Karman constant;

$\rho u^{\prime} w^{\prime}=$ shear stress;

$\sigma_{z}=$ standard deviation of the bed elevation; and

$\left\langle\tau_{0}\right\rangle=$ integral bed-shear stress.

\section{References}

Aberle, J. (2007). "Measurements of armor layer roughness geometry function and porosity." Acta Geophysica, 55(1), 23-32.

Aberle, J., and Nikora, V. (2006). "Statistical properties of armored gravel bed surfaces." Water Resour. Res., 42, W11414.

Bayazit, M. (1975). "Free surface flow in a channel of large relative roughness." J. Hydraul. Res., 14(2), 115-126.

Bhaganagar, K., Kim, J., and Coleman, G. (2004). "Effect of roughness on wall-bounded turbulence." Flow, Turbul. Combust., 72, 463-492.

Calhoun, R. J., and Street, R. L. (2001). "Turbulent flow over a wavy surface: Neutral case.” J. Geophys. Res., 106(C5), 9277-9293.

Carollo, F. G., Ferro, V., and Termini, D. (2005). "Analyzing turbulence intensity in gravel bed channels." J. Hydraul. Eng., 131(12), 10501061.

Cebeci, T., and Bradshaw, P. (1977). Momentum transfer in boundary layers, Hemisphere, Washington, D.C.

Cui, J., Patel, V. C., and Lin, C. L. (2003). "Large-eddy simulation of turbulent flow in a channel with rib roughness." Int. J. Heat Fluid Flow, 24(3), 372-388.

Dittrich, A., and Koll, K. (1997). "Velocity field and resistance of flow over rough surfaces with large and small relative roughness." Int. J. Sediment Res., 12(3), 21-33.

Germano, M., Piomelli, U., Moint, P., and Gebot, W. (1991). "A dynamic subgrid-scale eddy viscosity model." Phys. Fluids, 3(7), 1760-1765.

Grass, A. J. (1971). "Structural features of turbulent flow over smooth and rough boundaries." J. Fluid Mech., 50(2), 233-255.

Kironoto, B. A., and Graf, W. H. (1994). "Turbulence characteristics in rough uniform open-channel flow." Proc. Inst. Civ. Eng., Parts 1 and 2, 106(4), 333-344.

Lamb, M. P., Dietrich, W. E., and Venditti, J. G. (2008). "Is the critical shields stress for incipient sediment motion dependent on channel-bed slope?." J. Geophys. Res., 113, F02008.

Lee, C. (2002). "Large-eddy simulation of rough-wall turbulent boundary layers." AIAA J., 40(10), 2127-2130.

Leonardi, S., Orlandi, P., Smalley, R. J., Djenidi, L., and Antonia, R. A. (2003). "Direct numerical simulations of turbulent channel flow with transverse square bars on one wall." J. Fluid Mech., 491, 229-238.

Marsaglia, G., and Bray, T. A. (1964). "A convenient method for generating normal variables." SIAM Rev., 6, 260-264.

Moser, R. D., Kim, J., and Mansour, N. N. (1999). "DNS of turbulent channel flow up to $\mathrm{R} \tau=590$." Phys. Fluids, 11, 943-945.

Nakayama, A., Hori, K., and Street, R. L. (2004). "Filtering and LES of flow over irregular rough boundary." Proc., Summer Program 2004, Center Turbulence Research, Stanford, 145-156.

Nikora, V., and Goring, D. (2000). "Flow turbulence over fixed and weakly mobile gravel beds." J. Hydraul. Eng., 126(9), 679-690. 
Nikora, V., Goring, D., McEwan, I., and Griffiths, G. (2001). "Spatiallyaveraged open-channel flow over a rough bed." J. Hydraul. Eng., 127(2), 123-133.

Nikora, V., Koll, K., McEwan, I., McLean, S., and Dittrich, A. (2004). "Velocity distribution in the roughness layer of rough-bed flows." $J$. Hydraul. Eng., 130(10), 1036-1042.

Nikora, V., McEwan, I., McLean, S., Coleman, S., Pokrajac, D., and Walters, R. (2007). "Double-averaging concept for rough-bed openchannel and overland flows: Theoretical background." J. Hydraul. Eng., 133(8), 873-883.

Rahman, S., and Webster, D. (2005). "The effect of bed roughness on scalar fluctuations in turbulent boundary layers.” Exp. Fluids, 38(3), $372-384$.

Scotti, A. (2006). "Direct numerical simulation of turbulent channel flows with boundary roughened with virtual sandpaper." Phys. Fluids, 18, 031701.

Singh, K. M., Sandham, N. D., and Williams, J. J. R. (2007). "Numerical simulation of flow over a rough bed." J. Hydraul. Eng., 133(4), 386398.

Smagorinsky, J. (1963). "General circulation experiments with the primitive equations, Part I: The basic experiment." Mon. Weather Rev., 91, 99-152.
Song, T., Lemmin, U., and Graf, W. H. (1994). "Uniform flow in open channels with movable gravel bed." J. Hydraul. Res., 32(6), 861-876.

Stoesser, T., Braun, C., Garcia-Villalba, M., and Rodi, W. (2008). "Turbulence structures in flow over two dimensional dunes." J. Hydraul. Eng., 134(1), 42-55.

Stoesser, T., and Nikora, V. (2008). "Flow structure over square bars at intermediate submergence: Large-eddy simulation study of bar spacing effect." Acta Geophysica, 56(3), 876-893.

Tachie, M. F., Bergstrom, D. J., and Balachandar, R. (2000). "Rough wall turbulent boundary layers in shallow open channel flow." J. Fluids Eng., 122(3), 533-541.

Tachie, M. F., Bergstrom, D. J., and Balachandar, R. (2004). "Roughness effects on the mixing properties in open channel turbulent boundary layers." J. Fluids Eng., 126(6), 1025-1032.

Wang, J. C., Chen, C. K., Dong, Z. N., and Zhenhuan, X. (1993). "The effects of bed roughness on the distribution of turbulent intensities in open-channel flow." J. Hydraul. Res., 31(1), 89-98.

Wang, J. J., and Dong, Z. N. (1996). "Open-channel turbulent flow over nonuniform gravel beds.” Appl. Sci. Res., 56(4), 243-254.

Yue, W., Lin, C. L., and Patel, V. C. (2006). "Large-eddy simulation of turbulent flow over a fixed two-dimensional dune." J. Hydraul. Eng., 132(7), 643-651. 